\title{
INTEGRALIDADE DA ASSISTÊNCIA AO PACIENTE CIRÚRGICO: ARTICULAÇÃO ENTRE $O$ HOSPITAL E A ATENÇÃO PRIMÁRIA
}

DOI: 10.5327/Z1414-4425201900040001

0 modelo de atenção à saúde antes do Sistema Único de Saúde (SUS) era predominantemente individual, biomédico e centrado na doença. A abordagem da assistência à saúde ao paciente era realizada de forma compartimentalizada e pontual, focada no corpo biológico e na resolução dos sintomas apresentados. A instituição hospitalar, como representante máxima desse modelo, tinha lugar de destaque, tanto em relação à valorização da sua oferta de serviços quanto ao nível hierárquico que ocupava entre os equipamentos de saúde, retratando um sistema hierarquizado e fragmentado ${ }^{1}$.

Após a reforma sanitária, o entendimento de que saúde não é apenas a ausência de doença culminou com a criação do SUS, que adotou um modelo de atenção integral no qual o indivíduo é visto como um todo indivisível, e o processo de adoecimento, como resultado não apenas de disfunções anatomofisiológicas, mas, além disso, determinado por condições sociais, ambientais e afetadas pelas redes de apoio ${ }^{1}$.

Nesse sentido, as ações de promoção, proteção e recuperação da saúde também devem formar um todo indivisível e não podem ser compartimentalizadas, assim como as unidades prestadoras de serviço, com seus diversos graus de complexidade, devem estar configuradas em um sistema capaz de prestar assistência integral ao paciente ${ }^{1,2}$.

Para isso, é preciso garantir a continuidade do cuidado, por meio de um processo de transição adequado entre os pontos das redes de atenção à saúde, organizações poliárquicas com objetivos comuns norteadores de ofertas de serviços e coordenadas pela atenção primária² .

Com a mudança do perfil demográfico e o aumento das condições crônicas, o sistema de saúde é desafiado a se estruturar em um modelo integrado e cooperativo de serviços, que atenda às necessidades de saúde da população de forma integrada, com cuidados contínuos e monitorando a saúde ${ }^{2}$.

O paciente que é submetido a uma intervenção cirúrgica, após a alta médica, necessita de um processo de transição entre o ambiente hospitalar e o domiciliar livre de riscos e que assegure a continuidade da assistência e a preparação do ambiente e da família. Essa continuidade é fundamental para prevenir complicações, reinternações, reduzir gastos em saúde e promover a qualidade de vida do paciente e da família. A depender do grau de complexidade do paciente, essa transição pode ser um processo complicado, que exige dos profissionais adequada comunicação, conhecimento e habilidades ${ }^{3}$.

$\mathrm{Na}$ literatura, têm sido relatadas estratégias para o atendimento integral e a garantia da continuidade da assistência nesse processo de transição. Como exemplo, temos a experiência do sistema de saúde catalão, na Espanha, com as enfermeiras hospitalares de Enlace, cujo trabalho é verificar a necessidade de continuidade do cuidado após a alta, por meio de interconsulta com a equipe multiprofissional e de busca ativa no sistema de informação hospitalar dos pacientes internados. As enfermeiras têm conhecimento sobre os recursos necessários para realizar essa continuidade e travam contato com os profissionais da área de origem do paciente (cuidados primários, centros de saúde, hospital e/ ou residência) por telefone, e-mail ou por sistemas informatizados compartilhados entre os serviços, garantindo uma visita ao paciente, feita pelo enfermeiro ou médico da atenção primária, até 48 horas após a alta hospitalar ${ }^{4}$.

No Brasil, modelo semelhante ao espanhol é desenvolvido em uma região do município de São Paulo, onde existe a figura do gestor de casos, que articula o serviço hospitalar com as Unidades Básicas de Saúde (UBS) e com o serviço de assistência domiciliar. $\mathrm{O}$ gestor de casos, que pode ser um enfermeiro ou assistente social, identifica os pacientes com condições crônicas complexas na alta hospitalar, grupo que muitas vezes inclui os pacientes cirúrgicos, e realiza o encaminhamento para a UBS de referência, para acompanhamento. Caso necessário, faz a avaliação para admissão no serviço de assistência domiciliar imediatamente após a alta ${ }^{5}$. 
Outra iniciativa encontrada na literatura é um sistema de comunicação baseado em uma plataforma da internet, desenvolvido como uma rede social adaptada para o uso dos profissionais de saúde, que permite o compartilhamento de informações, documentos e planos de tratamento entre o hospital e as UBS, promovendo troca de informações e diálogos sobre a condição e o tratamento dos pacientes, durante e após as internações e reinternações ${ }^{6}$.

No tocante à estrutura pouco integrada e articulada do nosso sistema de saúde atual, precisamos nos integrar mais, assim como estamos fazendo aqui neste editorial. Outras ações incluem utilizar as ferramentas da atenção interprofissional colaborativa e centrada no paciente e promover a integração entre as disciplinas desde a formação, com currículos que não compartimentalizem o paciente em sistemas, os profissionais em especialidades e os serviços em ilhas especializadas de assistência.

Compreender a integralidade e a continuidade da assistência como fatores protetores e de promoção à saúde do paciente é tão fundamental para melhores resultados do tratamento quanto seguir a prescrição da alta médica e os cuidados de enfermagem.

Fernanda Amendola (1) Enfermeira, doutora em Ciências pela Universidade de São Paulo (USP). Professora dos cursos de graduação e pós-graduação da Faculdade Israelita de Ciências da Saúde Albert Einstein (FICSAE), São Paulo, Brasil.

\section{REFERÊNCIAS}

1. Fertonani HP, de Pires DEP, Biff D, Scherer MDA. Modelo assistencial em saúde: conceitos e desafios para a atenção básica brasileira. Ciênc Saúde Coletiva [Internet]. 2015 [acessado em 10 out. 2019];20(6):1869-78. Disponivel em: https://www. scielosp.org/pdf/csc/2015.v20n6/1869-1878/pt. http://doi. org/10.1590/1413-81232015206.13272014

2. Mendes EV. As redes de atenção à saúde. Ciênc Saúde Coletiva [Internet]. 2010 [acessado em 12 out. 2019];15(5):2297-305. Disponível em: http://www.scielo.br/scielo.php?script=sci_ arttext\&pid=S1413-81232010000500005\&lng=en. http://dx.doi. org/10.1590/S1413-81232010000500005

3. Weber LAF, Lima MADS, Acosta AM, Marques GQ. Transição do cuidado do hospital para o domicílio: revisão integrativa. Cogitare Enferm [Internet]. 2017 [acessado em 10 out. 2019];22(3):e47615. Disponível em: http://dx.doi.org/10.5380/ce.v22i3.47615
4. da Costa MFBNA, de Andrade SR, Soares CF, Pérez EIB, Tomás SC, Bernardino E. A continuidade do cuidado de enfermagem hospitalar para a Atenção Primaria à Saúde na Espanha. Rev Esc Enferm USP. 2019;53:e03477. http://dx.doi.org/10.1590/s1980-220x2018017803477

5. Duayer MFF. Análise de implantação de um programa de gestão de casos no cuidado de idosos frágeis na Atenção Primária à Saúde [tese]. São Paulo: Universidade de São Paulo; 2018. http://dx.doi. org/10.11606/T.83.2019.tde-08052019-172811

6. Bracco MM, Mafra ACCN, Abdo AH, Colugnati FAB, Dalla MDB, Demarzo MMP, et al. Implementation of integration strategies between primary care units and a regional general hospital in Brazil to update and connect health care professionals: a quasi experimental study protocol. BMCHealth Services Res [Internet]. 2016 [acessado em 22 set. 2017];16:380. Disponível em: https://www.ncbi.nlm.nih.gov/pmc/articles/PMC4983016/pdf/12913_2016_ Article_1626.pdf. http://dx.doi.org/10.1186/s12913-016-1626-9 